Revista Verde de Agroecologia e Desenvolvimento Sustentável

http://www.gvaa.com.br/revista/index.php/RVADS

ARTIGO CIENTÍFICO

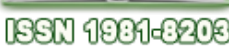

DOI: http://dx.doi.org/10.18378/rvads.v10i2.3475

\title{
Substratos alternativos na produção de mudas de flamboyant e ipê-mirim
}

\author{
Alternative substrates in the production of seedlings of flamboyant and ipe-mirim \\ Marília Dutra Massad ${ }^{1}$, Tiago Reis Dutra ${ }^{2}$, Tiago Barbosa Santos ${ }^{3}$, Rafaela Letícia Ramires Cardoso $^{4}$, Mateus Felipe $^{5}$
Quintino Sarmento
}

Resumo: O trabalho teve como objetivo avaliar o efeito do uso de bagaço de cana, casca de urucum e serragem, em diferentes proporções, na composição de substratos alternativos, constituídos a partir da mistura do substrato comercial Rohrbacherß, para a produção de mudas de flamboyant e ipê-mirim. Foi adotado o delineamento experimental de blocos ao acaso, com três repetições, no esquema fatorial $(5 \times$ 2), sendo avaliados cinco tipos de substratos e duas espécies. Os substratos avaliados foram: 100\% Rohrbacher®; 70\% Rohrbacher® + 30\% Bagaço de cana; 70\% Rohrbacher® + 30\% Casca de urucum; 70\% Rohrbacher ${ }^{\circledR}+30 \%$ Serragem; $50 \%$ Rohrbacher ${ }^{\circledR}+16,67 \%$ Bagaço de cana $+16,67 \%$ Casca de urucum $+16,67 \%$ Serragem . Foram avaliados: altura da parte aérea; diâmetro de coleto; massa seca da parte aérea; massa seca da raiz e massa seca total, além das relações H/DC, H/MSPA, MSPA/MSR e Índice de Qualidade de Dickson. Os substratos Rohrbacher®, $70 \% \mathrm{R}+30 \% \mathrm{CA}$ e $70 \% \mathrm{R}+30 \% \mathrm{UR}$ promoveram maior ganho em altura nas mudas de flamboyant e ipê-mirim. O substrato Rohrbacher® proporcionou às mudas maior produção de MSR. O flamboyant apresentou valores superiores para H, DC, MSPA, MSR, MST MSPA/MSR e IQD, conferindo um padrão de qualidade de mudas superior ao ipê-mirim.

Palavras-chaves: Delonix regia, resíduos orgânicos, silvicultura, Tecoma stans

\begin{abstract}
The study aimed to assess the effect of using sugarcane bagasse, bark and sawdust annatto, in different proportions, the composition of alternative substrates, made from a mixture of commercial substrate Rohrbacher@, to produce flamboyant and seedlings ipe-mirim. The experimental design of randomized blocks was adopted with three replications, in a factorial scheme (5 x 2), being evaluated five types of substrates and two species. The substrates were evaluated: $100 \%$ Rohrbacher®; Rohrbacher ${ }^{\circledR} 70 \%+30 \%$ Sugar cane bagasse; Rohrbacher ${ }^{\circledR} 70 \%+30 \%$ annatto bark; Rohrbacher ${ }^{\circledR} 70 \%+30 \%$ sawdust; $50 \%$ Rohrbacher ${ }^{\circledR}+16.67 \%$ Sugar cane bagasse $+16.67 \%$ annatto Bark $+16.67 \%$ sawdust. They were assessed: shoot height; diameter collect; dry mass of shoots; dry mass of roots and total dry matter, beyond relations H / DC, H / SDM, SDM / RDM and Quality Index of Dickson. The Rohrbacher® substrates, 70\% R +30\% CA and 70\% R + 30\% RH promoted greater height gain in seedlings flamboyant and ipe-mirim. The Rohrbacher ${ }^{\circledR}$ substrate provided the seedlings higher production of MSR. The flamboyant showed higher values for H, DC, SDM, RDM, TDM, SDM / SDM and QID, giving a quality standard of superior seedlings to ipe-mirim.
\end{abstract}

Key words: Delonix regia, organic waste, forestry, Tecoma stans

\footnotetext{
*Autor para correspondência

Recebido para publicação em 23/05/2015; aprovado em 30/06/2015

${ }^{1}$ Professora do Instituto Federal do Norte de Minas Gerais, Câmpus Salinas. Salinas-MG. E-mail: mariliamassad@yahoo.com.br

${ }^{2}$ Professor do Instituto Federal do Norte de Minas Gerais, Câmpus Salinas. Salinas-MG. E-mail: tiagoreisdutra@ gmail.com

${ }^{3}$ Engenheiro Florestal. E-mail: tiagojce@ hotmail.com

${ }^{4}$ Engenheira Florestal. E-mail: leh.floresta@yahoo.com.br

${ }^{5}$ Mestrando em Ciência Florestal, Universidade Federal dos Vales do Jequitinhonha e Mucuri, Câmpus Diamantina. E-mail: mateusengflorestal@ hotmail.com
} 


\section{INTRODUÇÃO}

A espécie Delonix regia, popularmente conhecida como flamboyant, flor-do-paraiso, pau-rosa, ponciana real e acaciarubra, é pertencente à família Fabaceae. Apresenta potencial paisagístico com flores atraentes e vistosas de tonalidades que variam desde alarajando-claro até vermelho-sanguíneo (SILVA et al., 2008). Extremamente florífera e ornamental, apresenta 10 a 12 metros de altura, sendo adequada para uso paisagístico em geral, na arborização urbana e ornamentação de parques, onde haja espaço suficiente para o seu desenvolvimento (LORENZI et al., 2003).

O ipê-mirim, espécie Tectona stans, é também conhecido como amarelinho e ipêzinho-de-jardim. É uma árvore de médio porte, apresentando de 4 a 6 metros de altura e $25 \mathrm{~cm}$ de diâmetro à altura do peito. No Brasil, devido à exuberância de suas flores de coloração amarelada durante todos os meses do ano, a espécie encontra-se como um dos melhores elementos de ornamentação para jardins e praças, para todas as regiões do país, exceto onde ocorram geadas muito severas, pois a espécie não é tolerante a baixas temperaturas (RENÓ, 2004; LORENZI et al., 2003.)

A obtenção de mudas de espécies utilizadas na arborização urbana como o flamboyant e o ipê-mirim apresentam desafios como, a produção de mudas sadias, de alta qualidade, em um menor intervalo de tempo e com menores custos.

Um dos meios sugeridos para superar esses obstáculos é a utilização de substratos alternativos elaborados a partir de resíduos orgânicos, que estejam regionalmente disponíveis e com grande facilidade de acesso.

A utilização dos resíduos orgânicos na composição dos substratos indica uma alternativa para a reciclagem de resíduos agroindustriais e industriais (CALDEIRA et al., 2013; NEVES et al., 2010), além da aquisição de misturas ideais que sirvam de suporte para o desenvolvimento das plantas.

Como exemplo de resíduos orgânicos para a composição de substratos alternativos facilmente encontrados na região de Salinas, no estado de Minas Gerais, tem o bagaço de cana, a casca de urucum e o pó de serragem, ambos testados em outras espécies, por diversos autores quanto à sua viabilidade no que diz respeito às características químicas e físicas (GONÇALVES et al., 2013; GOMES et at., 2010; DUTRA et al., 2013).

O cultivo do urucum é feito por muitos produtores da região e os resíduos da planta, como a sua casca são descartados em grande quantidade na época de colheita do fruto.

A serragem, embora seja utilizada como fonte energética e também na fabricação do MDF, não é integralmente aproveitada devido à grande quantidade gerada nos processos da serraria e também devido às grandes distâncias dos centros consumidores. A queima desse resíduo é uma prática comum e gera a emissão de gases tóxicos para a atmosfera, agravando o problema ambiental. Seu aproveitamento poderá reduzir gradativamente tais problemas, diminuir também o custo de produção agrícola e florestal, aliando a eficiência da produção.
O bagaço de cana, produto derivado da cana-de-açúcar é encontrado em abundância na região de Salinas-MG, considerada a maior produtora de cachaça do país.

Seu reaproveitamento na formulação de substratos demonstra uma excelente alternativa por se tratar de um resíduo amplamente disponível e por manter estáveis suas características físicas por um período suficientemente longo para que possa ser utilizado na produção de mudas (SILVA et al., 2008).

Desta forma, a utilização dos resíduos da agroindústria e da indústria, como componentes de substratos orgânicos, minimiza a incineração do material com posterior liberação de gases poluentes para a atmosfera, e ainda o descarte a céu aberto ou em aterros sanitários, contribuindo para diminuir o seu acúmulo no meio ambiente (MOREIRA et al., 2011).

Assim, obtém-se um material alternativo, que apresenta baixo custo e disponível na região, que beneficia a reciclagem de nutrientes, melhora o desenvolvimento e por consequência a produtividade das culturas e torna os sistemas agrícolas mais sustentáveis. Além disso, diminui os custos de produção de mudas por substituir parcialmente a utilização do subtrato comercial.

O substrato deve fornecer as melhores condições para que haja excelente germinação e favoreça o desenvolvimento das mudas. Ele é considerado um dos fatores internos de maior influência no processo de enraizamento e qualidade das raízes formadas tendo assim papel crucial na sobrevivência inicial da planta (HOFFMANN et al., 2001). Assim, o substrato deve possuir boa capacidade de retenção de água, volume ótimo de espaços porosos preenchidos por gases e adequada taxa de difusão de oxigênio necessária à respiração das raízes, além de apresentar fácil disponibilidade de aquisição e transporte, ausência de patógenos, riqueza em nutrientes essenciais, textura e estrutura adequada (SILVA et al., 2001). O uso de um substrato inadequado pode ocasionar irregularidade ou até mesmo nulidade na germinação, logo, o substrato se constitui num dos fatores mais complexos na produção de mudas (ARAÚJO; SOBRINHO, 2011).

Apesar de amplamente testados, estudos que avaliem a eficiência desses materiais na composição de substratos alternativos para as espécies de flamboyant e ipê-mirim são inexistentes e a necessidade de realização de testes de viabilidade se faz de extrema importância como forma de disseminar o conhecimento a respeito dos efeitos de diferentes substratos, sob formulações para utilização nas espécies em destaque.

O trabalho tem como objetivo avaliar o efeito do uso de materiais alternativos como bagaço de cana, casca de urucum e serragem, em diferentes proporções, na composição de substratos alternativos, constituídos a partir da mistura do substrato comercial Rohrbacher®, para a produção de mudas de flamboyant e ipê-mirim.

\section{MATERIAL E MÉTODOS}

O trabalho foi conduzido no "Viveiro de Produção de Mudas Florestais" do Instituto Federal de Educação, Ciência e Tecnologia do Norte de Minas Gerais (IFNMG), Câmpus Salinas.

Foi adotado o delineamento experimental de blocos ao acaso, com três repetições, no esquema fatorial (5 x 2), 
sendo avaliados cinco tipos de substratos e duas espécies, flamboyant e ipê-mirim. A unidade experimental foi constituída por 12 mudas.

Os substratos avaliados foram: $100 \%$ substrato comercial Rohrbacher® (vermiculita, fibra de côco, cascas de pinus carbonizada, calcário e NPK) (100R); 70\% Rohrbacher® + 30\% Bagaço de cana $(70 \mathrm{R}+30 \mathrm{CA}) ; 70 \%$ Rohrbacher® + 30\% Casca de urucum (70R+30UR); 70\% Rohrbacher ${ }^{\circledR}+30 \%$ Serragem $\quad(70 \mathrm{R}+30 \mathrm{SE}) ; 50 \%$ Rohrbacher® $+16,67 \%$ Bagaço de cana $+16,67 \%$ Casca de urucum $+$

$16,67 \%$

Serragem

$(50 \mathrm{R}+16,67 \mathrm{CA}+16,67 \mathrm{UR}+16,67 \mathrm{SE})$.

As sementes de flamboyant e ipê-mirim foram coletadas de sete matrizes de cada espécie estudada, localizadas no município de Salinas - MG.

A caracterização química dos substratos foi realizada pelo Laboratório de Fertilidade do Solo da UFMG (Tabela 1). As características físicas de porosidade total, macroporosidade, microporosidade e capacidade máxima de retenção de água dos substratos, foram determinadas pela metodologia proposta por Carvalho e Silva (1992) (Tabela 1).

Tabela 1. Características químicas e físicas dos substratos utilizados na produção de mudas de flamboyant e ipê-mirim

\begin{tabular}{|c|c|c|c|c|c|}
\hline \multirow{2}{*}{ Características ${ }^{1}$} & \multicolumn{5}{|c|}{ Substrato $^{2}$} \\
\hline & $100 \mathrm{R}$ & $70 \mathrm{R}+30 \mathrm{CA}$ & 70R+30UR & 70R+30SE & $\mathrm{R}+\mathrm{CA}+\mathrm{UR}+\mathrm{SE}$ \\
\hline pH, água & 6,0 & 5,3 & 6,0 & 6,0 & 6,1 \\
\hline M.O, dag kg-1 & 15,63 & 5,84 & 19,34 & 14,60 & 10,82 \\
\hline $\mathrm{P}, \mathrm{mg} \mathrm{dm}^{-3}$ & 260,0 & 168,8 & 380,0 & 260,0 & 220,0 \\
\hline $\mathrm{K}, \mathrm{mg} \mathrm{dm}^{-3}$ & 970 & 580,1 & 945,0 & 647 & 846 \\
\hline $\mathrm{Ca}, \mathrm{cmol}_{\mathrm{c}} \mathrm{d} \mathrm{m}^{-3}$ & 5,10 & 3,13 & 4,10 & 3,00 & 3,00 \\
\hline $\mathrm{Mg}, \mathrm{cmol}_{\mathrm{c}} \mathrm{d} \mathrm{m}^{-3}$ & 1,60 & 1,03 & 1,50 & 1,20 & 1,40 \\
\hline $\mathrm{Al}, \mathrm{cmol}_{\mathrm{c}} \mathrm{d} \mathrm{m}^{-3}$ & 0,00 & 0,09 & 0,00 & 0,10 & 0,00 \\
\hline $\mathrm{H}+\mathrm{Al}, \mathrm{cmol}_{\mathrm{c}} \mathrm{d} \mathrm{m}^{-3}$ & 2,19 & 1,57 & 2,22 & 2,32 & 2,29 \\
\hline $\mathrm{t}, \mathrm{cmol}_{\mathrm{c}} \mathrm{d} \mathrm{m}^{-3}$ & 9,19 & 5,77 & 8,02 & 5,96 & 6,57 \\
\hline $\mathrm{T}, \mathrm{cmol}_{\mathrm{c}} \mathrm{d} \mathrm{m}^{-3}$ & 11,38 & 5,23 & 10,24 & 8,18 & 8,86 \\
\hline $\mathrm{SB}, \mathrm{cmol}_{\mathrm{c}} \mathrm{d} \mathrm{m}^{-3}$ & 9,19 & 8,44 & 8,02 & 5,86 & 6,57 \\
\hline $\mathrm{m}, \%$ & 0 & 2 & 0 & 2 & 0 \\
\hline $\mathrm{V}, \%$ & 81 & 78 & 78 & 72 & 74 \\
\hline Porosidade Total, \% & 61,19 & 67,59 & 70,32 & 79,73 & 74,36 \\
\hline Macroporosidade, $\%$ & 31,43 & 34,78 & 35,76 & 44,39 & 40,29 \\
\hline Microporosidade, \% & 29,76 & 32,81 & 34,56 & 35,34 & 34,07 \\
\hline CMRA, mL $55 \mathrm{~cm}^{-3}$ & 23,11 & 11,25 & 10,98 & 8,68 & 9,01 \\
\hline
\end{tabular}

${ }^{1}$ M.O. = matéria orgânica; $\mathrm{t}=$ capacidade efetiva de troca de cátions; $\mathrm{T}=$ capacidade de troca de cátions; $\mathrm{SB}=$ soma de bases; $\mathrm{m}=$ saturação por alumínio; $\mathrm{V}=$ saturação por bases; CMRA = Capacidade máxima de retenção de água. ${ }^{2} \mathrm{R}=\%$ de Rohrbacher ${ }^{\circledR} ; \mathrm{CA}=\%$ de Bagaço de Cana; UR $=\%$ de casca de Urucum; $\mathrm{SE}=\%$ de Serragem.

O bagaço de cana e a casca de urucum foram triturados em moinho desintegrador, em seguida, passados em peneira de malha de $6 \mathrm{~mm}$. A serragem utilizada no presente trabalho foi doada por uma serraria da cidade.

Os substratos foram preparados seguindo as proporções dos tratamentos propostos e após formulados foram adubados com 7,0 $\mathrm{g} \mathrm{dm}^{-3}$ de Osmocote $\circledR$ MiniPrill Controlled Realise 19-06-10, com tempo estimado de liberação entre 3 a 4 meses. Em seguida, foram utilizados para preencherem os tubetes com capacidade volumétrica de $180 \mathrm{~cm}^{3}$.

As sementes de flamboyant tiveram a quebra de sua dormência realizada por meio do método de imersão em água quente à $80^{\circ} \mathrm{C}$ por 5 minutos (ZWIRTES et al., 2013). Em seguida, juntamente, com as sementes de ipê-mirim foram desinfestadas em solução de hipoclorito de sódio (2\%) por 3 minutos, posteriormente lavadas em água corrente e dispostas em um número de 3 sementes por tubete.

Aos 15 dias após semeadura (DAS) foi efetuado um primeiro raleio deixando-se duas plantas por tubete. Aos 30 DAS um segundo raleio foi realizado, deixando-se apenas uma muda por tubete.

A partir do $40^{\circ} \mathrm{DAS}$, as mudas receberam fertirrigação semanal, com $6 \mathrm{~mL}$ planta $^{-1}$ de solução aquosa, contendo $4 \mathrm{~g}$ $\mathrm{L}^{-1}$ de sulfato de amônio, $10 \mathrm{~g} \mathrm{~L}^{-1}$ de superfosfato simples, $4 \mathrm{~g}$ $\mathrm{L}^{-1}$ de cloreto de potássio e $1 \mathrm{~g} \mathrm{~L}^{-1}$ de FTE BR12 (9\% Zn, 3\% $\mathrm{Fe}, 2 \% \mathrm{Mn}, 0,1 \% \mathrm{Mo}, 1,8 \% \mathrm{~B}, 0,8 \% \mathrm{Cu}$ ).
Durante o período experimental, a umidade do solo foi mantida próxima da capacidade de campo, procedendo-se o monitoramento diário para esse controle.

Foram avaliados aos 120 dias a altura $(\mathrm{H} ; \mathrm{cm})$ e o diâmetro do coleto (DC; mm) das mudas. A mensuração da altura da parte aérea foi realizada com o auxílio de uma régua milimetrada posicionada no nível do substrato até o meristema apical das mesmas. O diâmetro foi medido por meio do uso de um paquímetro digital.

Posteriormente, as plantas foram colhidas e separadas em parte aérea e sistema radicular, lavadas em água corrente e secas em estufa com circulação forçada de ar, a aproximadamente $65^{\circ} \mathrm{C}$, até peso constante. Foi avaliada a massa seca da parte aérea (MSPA; g planta $^{-1}$ ), massa seca da raiz (MSR; $g$ planta $\left.{ }^{-1}\right)$ e massa seca total $($ MST $=$ MSPA + MSR; g planta $\left.{ }^{-1}\right)$.

Esses parâmetros foram transformados em índices de qualidade de mudas conforme sugerido por Gomes et al. (2002): H/DC, H/MSPA, MSPA/MSR e no Índice de Qualidade de Dickson-IQD (DICKSON et al., 1960), calculado por:

$$
I Q D=M S T(\mathrm{~g}) /[H(\mathrm{~cm}) / D C(\mathrm{~mm})+\operatorname{MSPA}(\mathrm{g}) / \operatorname{MSR}(\mathrm{g})
$$

Os dados obtidos foram submetidos à análise de variância e, quando o efeito do tipo de substrato em relação às especies foi significativo, as médias foram comparadas pelo 
teste de Tukey $(\mathrm{p}<0,05)$. Todas as análises estatísticas foram realizadas com o software Statistica 8.0.

\section{RESULTADOS E DISCUSSÃO}

Não houve efeito significativo da interação entre as duas espécies estudadas e os tipos de substratos para os parâmetros avaliados, ocorrendo somente o efeito isolado desses fatores para altura (H) e massa seca da raiz (MSR) até os 120 DAS.

A ausência de efeito significativo dos tipos de substratos para os parâmetros diâmetro de coleto, massa seca da parte aérea, massa seca total e Índice de Qualidade de Dickson, permite a possibilidade de uso de qualquer um dos substratos estudados para a produção de mudas de flamboyant e ipêmirim até os 120 DAS.

Observa-se que os substratos Rohrbacher®, $70 \% \mathrm{R}+30 \% \mathrm{CA}$ e $70 \% \mathrm{R}+30 \% \mathrm{UR}$ promoveram maior ganho em altura nas mudas, se diferenciando estatisticamente dos demais tratamentos estudados (Figura 1). Nota-se que o acréscimo do bagaço de cana e da casca de urucum ao substrato comercial para a composição do $70 \% \mathrm{R}+30 \% \mathrm{CA}$ e $70 \% \mathrm{R}+30 \% \mathrm{UR}$ manteve próximas as características físicas e químicas dos mesmos, quando comparados ao substrato comercial, favorecendo o desenvolvimento das mudas (Tabela 1).

Figura 1. Altura e massa seca da raiz das mudas cultivadas em cinco tipos de substratos.

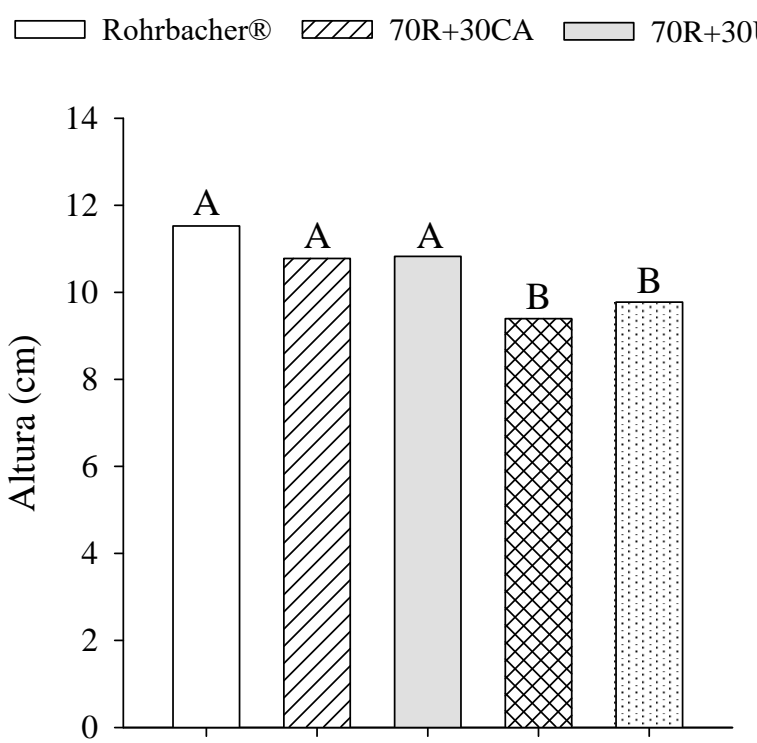

Substrato

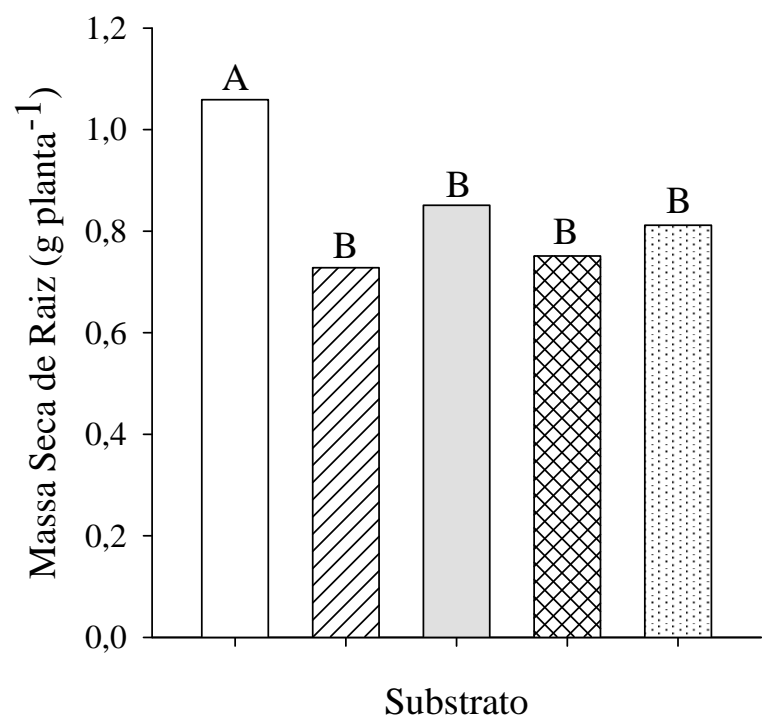

Para o parâmetro massa seca da raiz o substrato comercial Rohrbacher® apresentou as maiores médias, diferenciando-se estatisticamente aos demais substratos estudados (Figura 1). Observa-se que esse substrato apresentou a maior capacidade de retenção de água $(23,11 \mathrm{~mL}$ $\left.\left(55 \mathrm{~cm}^{-3}\right)^{-1}\right)$ possivelmente suprindo melhor a demanda da espécie por água. Segundo Gonçalves e Poggiani (1996), o valor adequado da CMRA no substrato deve estar entre 20 a $30 \mathrm{~mL}\left(55 \mathrm{~cm}^{-3}\right)^{-1}$. Nos demais tratamentos esse valor foi inferior (Tabela 1), o que pode ter prejudicado o desenvolvimento das plantas, refletindo no menor crescimento das raízes.

Menores valores de massa seca da raiz em substratos alternativos também foram observados nas espécies sucará (Gleditschia amorphoides), angico vermelho (Anadenanthera macrocarpa) e cedro rosa (Cedrela odorata) (BORTOLINI et al., 2012; ULIANA, 2009).

O flamboyant obteve valores superiores para os parâmetros H, DC, MSPA, MSR, MST, MSPA/MSR e IQD quando comparado ao ipê-mirim. O ipê mirim se destacou em H/DC e H/MSPA (Tabela 2). 
Tabela 2. Valores médios de altura, diâmetro do coleto, massa seca da parte aérea (MSPA), massa seca de raiz (MSR), massa seca total (MST), relação entre altura e diâmetro do coleto (H/DC), relação entre altura e massa seca da parte aérea (H/MSPA), relação entre a massa seca da parte aérea e massa seca da raiz (MSPA/MSR) e Índice de Qualidade de Dickson (IQD) das mudas de flamboyant e ipê- mirim aos 120 dias

\begin{tabular}{|c|c|c|c|c|c|c|c|c|c|}
\hline \multirow[t]{2}{*}{ Espécie $^{1}$} & \multicolumn{9}{|c|}{ Variáveis } \\
\hline & $\begin{array}{c}\text { Altura } \\
\mathrm{cm}\end{array}$ & $\begin{array}{c}\text { Diâmetro } \\
\text { mm }\end{array}$ & MSPA & $\begin{array}{c}\text { MSR } \\
\text { planta }^{-1}\end{array}$ & MST & $\mathrm{H} / \mathrm{DC}$ & H/MSPA & MSPA/MSR & IQD \\
\hline Flamboyant & $11,70 \mathrm{a}$ & $6,35 \mathrm{a}$ & $1,26 \mathrm{a}$ & $1,02 \mathrm{a}$ & $2,28 \mathrm{a}$ & $1,84 \mathrm{~b}$ & $9,28 \mathrm{~b}$ & $1,24 \mathrm{a}$ & $0,56 \mathrm{a}$ \\
\hline Ipê-mirim & $9,20 \mathrm{~b}$ & $3,28 \mathrm{~b}$ & $0,60 \mathrm{~b}$ & $0,66 \mathrm{~b}$ & $1,26 \mathrm{~b}$ & $2,80 \mathrm{a}$ & $15,33 \mathrm{a}$ & $0,91 \mathrm{~b}$ & $0,50 \mathrm{~b}$ \\
\hline
\end{tabular}

${ }^{1}$ Valores seguidos de letras distintas na mesma coluna diferem entre si pelo teste $\mathrm{F}$.

O flamboyant pertence à família Fabaceae, tendo por característica a rápida germinação e crescimento, rusticidade, grande capacidade fotossintética, vigor e porte, com altura de 10 a 12 metros (LORENZI et al., 2003; SILVA et al., 2008). Além disso, ele apresenta raízes pivotante e laterais bastante agressivas, caracterizadas como tabulares, tornando-a própria para arborização em praças (CHAVES et al., 2013; TOSCAN et al., 2010). Essas características conferem à espécie um desenvolvimento superior para os parâmetros em que ela se destacou quando comparado ao ipê-mirim. Observou-se também médias superiores nas mudas de flamboyant para MSPA, MSR e MST (Tabela 2). Esses valores estão relacionados com as características da espécie descritas anteriormente. O peso da MSPA é um importante parâmetro para indicar a rusticidade, melhor vigor e correlaciona-se diretamente com o desempenho inicial das mudas e a sobrevivência no campo após o plantio (CRUZ et al., 2010).

Observou-se que o flamboyant obteve valores médios de MSPA/MSR maiores $(1,24)$ em comparação ao ipê-mirim $(0,94)$ (Tabela 2), alcançando assim um padrão de qualidade de mudas melhor. Entretanto, Brissette (1984), citado por Cruz et al. (2010), menciona que o valor 2,0 é considerado a melhor relação entre o MSPA/MSR de uma mesma planta, pois esse valor representa um maior equilíbrio entre a parte aérea e raiz da muda. Observa-se que os valores das médias das duas espécies estudadas estavam abaixo do ideal, assim, pode-se presumir que essas mudas deveriam ter permanecido mais tempo no viveiro.

No parâmetro H/DC, o ipê-mirim obteve valores médios maiores em relação ao flamboyant (Tabela 2). Esse fato se deve em função da mudas apresentarem-se mais delgadas. Para H/MSPA o ipê-mirim obteve valores médios maiores em comparação ao flamboyant, o que nos mostra que o flamboyant se desenvolveu melhor, devido ao fato que quanto menor for a relação entre a altura e a massa seca da parte aérea, mais lignificada está a muda e maior a sua capacidade de sobrevivência no campo (GOMES et al., 2002).

O Índice de Qualidade de Dickson encontrado nas mudas de flamboyant foi superior às mudas de ipê-mirim (0,56 e 0,50, respectivamente) (Tabela 2). Desta forma, o flamboyant apresentou um melhor padrão de qualidade da muda. Entretanto, as duas espécies estudadas apresentaram médias superiores ao valor mínimo de 0,20 , recomendado na classificação de qualidade de mudas (HUNT, 1990, citado por GOMES et al., 2002).

\section{CONCLUSÕES}

Os substratos Rohrbacher®, $70 \% \mathrm{R}+30 \% \mathrm{CA}$ e $70 \% \mathrm{R}+30 \% \mathrm{UR}$ promoveram maior ganho em altura nas mudas de flamboyant e ipê-mirim. O substrato Rohrbacher® proporcionou às mudas maior produção de MSR.

O flamboyant apresentou valores superiores para H, DC, MSPA, MSR, MST MSPA/MSR e IQD, o que lhe conferiu um padrão de qualidade de mudas superior ao ipê-mirim.

\section{REFERÊNCIAS BIBLIOGRÁFICAS}

ARAÚJO, A. P. de; SOBRINHO, S. de P. Germinação e produção de mudas de tamboril (Enterolobium contortisiliquum (vell.) morong) em diferentes substratos. Revista Árvore, Viçosa, v.35, n.3, Edição Especial, p.581-588, 2011.

BORTOLINI, M. F.; KOEHLER, H. S.; ZUFFELLATORIBAS, K. C.; FORTES, A. M. T. Crescimento de mudas de Gleditschia amorphoides Taub. produzidas em diferentes substratos. Revista Ciência Florestal, Santa Maria, v.22, n.1, p.35-46, 2012.

CALDEIRA, M. V. W.; DELARMELINA, W. M.; FARIA, J. C. T.; JUVANHOL, R. S. Substratos alternativos na produção de mudas de Chamaecrista desvauxii. Revista Árvore,Viçosa, v.37, n.1, p.31-39, 2013.

CARVALHO, C. M.; SILVA, C. R. Determinação das propriedades físicas de substrato. Botucatu: Faculdade de Ciências Agronômicas: Universidade Estadual Paulista, 1992. 6p.

CHAVES, A. M. S.; SILVA, A. dos S.; AMADOR, M. B. M. Ausência de sincronia entre planejamento e a arborização urbana: um estudo de caso na avenida Rui Brabosa em Garanhuns - PE. Revista Nacional de Gerenciamento de Cidades, v. 1, n. 3, p. 54-71, 2013.

CRUZ C. A. F.; PAIVA, H. N. de; NEVES, J. C. L.; CUNHA, A. C. M. C. M. da. Resposta de mudas de Senna macranthera (Dc. ex collad.) H. S. Irmin \& Barnaby (Fedegoso) cultivadas em latossolo vermelho-amarelo distrófico a macronutrientes. Revista Árvore, Viçosa, v. 34, n. 1, p. 13-24, 2010.

DICKSON, A.; LEAF, A, L.; HOSNER, J. F. Qualitty appraisal of white spruce and white pine seedling stock in nurseries. Forestry Chronicle, v.36, p.10-13, 1960.

DUTRA, T. R., MASSAD, M. D.; SARMENTO, M. F. Q.; OLIVEIRA, J. C. de. Substratos alternativos e métodos de quebra de dormência para produção de mudas de canafístula. Revista Ceres, Viçosa, v. 62, p. 72-78, 2013. 
GOMES, J. M.; COUTO, L.; LEITE, H. G.; XAVIER, A.; GARCIA, S. L. R. parâmetros morfológicos na avaliação da qualidade de mudas de Eucalyptus grandis. Revista. Árvore, Viçosa, v.26, n.6, p.655-664, 2002.

GOMES, K. B. P.; VILARINO, M. de L. G.; SILVA, V. P.; FERRARO, A. C. Avaliação da emergência e do crescimento inicial de plântulas de cedro-rosa em diferentes substratos. Revista Agrogeoambiental, v.2, n.1, p.75-84, 2010.

GONÇALVES, J. L. M.; POGGIANI, F. Substratos para produção de mudas forestais. In: CONGRESSO LATINO AMERICANO DE CIÊNCIA DO SOLO, 13., 1996. Águas de Lindóia. Anais... Águas de Lindóia: USP ESALQ/SBCS/CEA/SLACS/SBM, 1996. 1 CD-ROM

GONÇALVES, F. G.; ALEXANDRE, R. S.; SILVA, A. G. da; LEMES, E. de Q.; ROCHA, A. P. da; RIBEIRO, M. P. de A. Emergência e qualidade de mudas de Enterolobium contortisiliquum (Vell.) Morong (Fabaceae) em diferentes substratos. Revista Árvore, Viçosa, v.37, n.6, 2013.

HOFFMANN, I.; GERLING, D.; KYIOGWOM, U. B.; MANÉ-BIELFELDT, A. Farmers management strategies to maintain soil fertility in a remote area in northwest Nigeria. Agriculture, Ecossystems \& Environment, v.86, p.263-275, 2001.

LORENZI, H.; SOUZA, H. M. de; TORRES, M. A. V.; BACHER, L. B. Árvores exóticas no Brasil: madeireiras, ornamentais e aromáticas. Nova Odessa: Instituto Plantarum, 2003. 368p.

MOREIRA, R. A.; RAMOS, J. D.; ARAÚJO, N. A. de; MARQUES, V. B. Produção e qualidade de frutos de pitaia-vermelha com adubação orgânica e granulado bioclástico. Revista Brasileira de Fruticultura, Jaboticabal, volume especial, p. 762-766, 2011.

NEVES, J. M. G.; SILVA, H. P. da; DUARTE, R. F. Uso de subtratos alternativos para produção de mudas de moringas. Revista Verde, Mossoró, v.5, n.1, p. 173-177, 2010.

RENÓ, L. R. Propagação vegetativa e fenologia de Tecoma stans (L.) JUSS. EX. KUNTH (BIGNONIACEAE). In. PEDROSA-MACEDO, J. H.; BREDOW, E. A. (Eds.). Princípios e rudimentos do controle biológico de plantas: coletânea. Curitiba: UFPR, 2004. p.107-111.

SILVA, R. P. da; PEIXOTO, J. R.; JUNQUEIRA, N. T. V. Influência de diversos substratos no desenvolvimento de mudas de maracujazeiro azedo (Passiflora edulis Sims f. flavicarpa DEG). Revista Brasileira de Fruticultura, Jaboticabal, v. 23, n. 2, p. 377-381, ago. 2001.

SILVA D. S. da; SPIER, M.; SOUZA, P. V. D. de; SCHÄFER, G. Caracteristicas químicas do bagaço de cana-de-açucar para uso como substrato para plantas . XX Congresso Brasileiro de Fruticultura 54th Annual Meeting of the Interamerican Society for Tropical Horticulture Outubro de 2008-Vitória-ES (Anais...). Disponível em <http://www.ufrgs.br/agronomia/materiais/ 683551.pdf $>$. Acesso em 19 jul 2015.

TOSCAN, M. A. G., RICKLI, H. C.; BARTINICK, D. SANTOS, D. S. dos; ROSSA, D. Inventário e análise da arborização do bairro Vila Yolanda, do município de Foz do Iguaçu - PR. Revista Revsbau, Piracicaba, v. 5, n. 3 , p. $165-184,2010$.

ULIANA, M. B. Substratos e fertirrigação na produção de mudas de Anadenanthera macrocarpa (Benth) Brenan e Cedrela odorata L. Dissertação de Mestrado. Universidade Estadual do Oeste do Paraná, Marechal Rondon, 2009. 72p

ZWIRTES, A. L.; BARONIO, C. A.; CANTARELLI, E. B.; RIGON, J. P. G.; CAPUANI, S. Métodos de superação de dormência em sementes de flamboyant. Pesquisa Florestal Brasileira, Colombo, v. 33, n. 76, p. 467-471, 2013. 